based on studies including the long-term effects of the different BMI cut-offs during childhood. Until the adoption of an international consensus the simultaneous use of different definitions will enlarge the possibilities of comparisons between studies. Such a consensus is urgently needed.

\title{
The role of the GP in prevention, management and treatment of overweight children: a 'big' problem
}

\author{
Andre Franck
}

Member of the 'High Council for medical specialists and family practioners' Federal Ministery of Health and Board Member Societe Belge Medecins Nutrionistes/Belgische Vereniging Artsen Voedingsdeskun Digen

The percentage of overweight children in the USA is growing at an alarming rate, with more than one out of three children now considered overweight, obese or even severely obese. The same trend is seen in Europe but with less-alarming statistics. Will the overwhelming epidemic of child obesity reach Europe? The most recent figures and statistics confirm the possibility. The last figures for Belgium from the 'Nationaal Voedings- en Gezondheidsplan 2006-2010' show that 18\% of children between 2 and 17 years are overweight and 5\% are obese, with the condition being most prevalent in the age group of 5-9 years. In this group 18\% are found to be obese!

Why do we have such an epidemic situation? What is the origin of better which are the origins of this epidemic? What is the cause or which are the causes? Is it only a question of weight or is it a more complex problem? Can obese children be classified into different groups and do they have different health conditions? When is a child considered overweight, obese or even severely obese? The age- and sex-adjusted BMI helps to determine the weight of the child. We find six categories pertaining to weight, ranging from underweight $(<5$ th percentile) to severe obesity (120th percentile and higher). BMI is not a perfect measure of body fat and can be misleading. It is a good indicator but not an exact measurement. Therefore, we need more sophisticated instruments (bioelectric impedance) to determine obesity. BMI may be difficult to interpret during puberty when children experience rapid growth. What are the effects of child obesity? Obesity increases the risk of serious health conditions and leads to marked changes in lifestyle and behaviour. Medical complications that manifest include CVD (hypertension, high cholesterol and so on), diabetes, bone and joint problems, tendency to mature earlier and so on. Behavioural, lifestyle and psychological complications include unhealthy dietary habits, eating disorders, depression and so on.

Causes of obesity: Obesity, which is becoming a serious epidemic worldwide, is a chronic disease prevalent among both children and adults, with a very complex physiopathology and involving many health complications.

Obesity leads to genetic and physiological transformations at three levels: in the cerebrum (neuroendocrine changes), in fat cells (adipokines) and in hormones in the gastroenteral tract.

Lifestyle habits play an important role in the spread of the obesity epidemic through change in eating habits, lack of exercise and overuse of electronic devises. Preventing overweight and obesity: Preventing kids from becoming overweight means changing the way the family eats and exercises and how they spend time together. Helping kids lead a healthy lifestyle begins with parents leading by example. Many kids are spending less time exercising and more time watching television and playing video games. Moreover, in the present day, families are becoming increasingly busy and devote less time to preparing nutritious meals. From fast food to electronics, quick and easy is the way of life for many people in the 21st century.

Not only the community but the individual practitioner also should play an active role in ensuring a healthy lifestyle in order to arrest the spread of obesity by taking the following steps:

- not succumbing to common food/eating traps;

- give recommendations by age;

- control BMI and undergo an annual clinical examination.

Treatment of child obesity: The AMA recommends a 4-stage approach that can be adapted to the situation in Europe.

Stage 1 - Prevention Plus Protocol

Stage 2 - Structured Weight Management Protocol

Stage 3 - Comprehensive Multidisciplinary Intervention

Stage 4 - Tertiary Care Intervention.

Many of these recommendations can be carried out by the family physicians and or by paediatricians.

However, Belgian paediatricians do not believe that treating obesity will give a good result in the long term! There is still much work to do. 